\title{
Metodología Alternativa para el Aprendizaje de la Dinámica de Fluidos, Caso ESIQIE
}

\author{
Fabiola Escobar Moreno, Maestra en Ingeniería \\ Rosa G. Nava Galve, Maestra en Ciencia de Materiales \\ ESIQIE del Instituto Politécnico Nacional, México
}

\section{Resumen}

Se propone una metodología alternativa para el aprendizaje de la dinámica de los fluidos, que contempla etapas y tiempos diferentes, comparada con otras metodologías (tradicional y Física recreativa + resolución de ejercicios+ problema de la Industria Química). Donde sobresale una aplicación de la dinámica de fluidos en el contexto de la industria química. El objetivo de la indagación es comprobar si la propuesta metodológica Física en el Contexto de la Ingeniería Química, mejora el aprendizaje de los alumnos para el tópico dinámica de fluidos. Lo anterior obedece a las variadas situaciones del aprendizaje de la dinámica de fluidos de los alumnos y que esto incide en su rendimiento académico, relacionado con unidades de aprendizaje (UA) de la Academia de Física de la ESIQIE (Escuela Superior de Ingeniería Química e Industrias Extractivas). De ahí, nuestra preocupación como docentes para contribuir al desarrollo de metodologías que permitan aprendizaje significativo y útil para los estudiantes, y así, en esa medida mejorar la calidad educativa en la mencionada Academia. La metodología de esta indagación es cuantitativa, basada en una prueba paramétrica ANOVA, el estadístico Factor de Hake; también, se utiliza estadística descriptiva basada en un instrumento relacionado con calidad en el servicio. Se evidencia a través de los resultados estadísticos que si hay diferencias significativas con la metodología Física en el Contexto de la Ingeniería Química.

Palabras claves: Alternativa metodológica, dinámica de fluidos, industria química, calidad en los servicios educativos 


\title{
Alternative Methodology for the Learning of Fluid Dynamics, ESIQIE Case
}

\author{
Fabiola Escobar Moreno, Maestra en Ingeniería \\ Rosa G. Nava Galve, Maestra en Ciencia de Materiales \\ ESIQIE del Instituto Politécnico Nacional, México
}

\begin{abstract}
An alternative methodology is proposed for the learning of fluid dynamics, which contemplates different stages and times, compared with other methodologies (traditional and recreational physics + resolution of exercises + problem of the Chemical Industry.) Where an application of fluid dynamics stands out in the context of the chemical industry, the objective of the investigation is to check whether the methodological proposal improves the learning of the students for the dynamic topic of fluids, which is due to the different learning situations of fluid dynamics of students and that this affects their academic performance, related to learning units of the Physics Academy of the ESIQIE (Escuela Superior de Ingeniería Química e Industrias Extractivas). Teachers to contribute to the development of methodologies that allow meaningful learning and useful for students, and thus, to that extent improve the quality of education in the Physical Academy. The methodology of this inquiry is quantitative, based on a parametric ANOVA test, the Hake Factor statistic; also, descriptive statistics based on an instrument related to quality in the service. It is evidenced through the statistical results that there are significant differences with the Physical methodology in the context of Chemical Engineering.
\end{abstract}

Keywords: Methodological alternative, fluid dynamics, chemical industry, quality in educational services

\section{Introduction}

Las obras clásicas de cursos introductorios de Física se han reinventado, porque, les inquieta mejorar la accesibilidad de la comprensión de la Física; aunque, siendo incisivos quizá también obedezca a razones de tipo económico y la ingente competencia entre editoriales y recursos en línea. Como ejemplo, está el renombrado libro de David Halliday y Robert Resnick, que conocedores de que su obra a más de 40 años de su publicación tiene que evolucionar; ya que, si no lo hacen tienden a perder usuarios y lectores. Así, 
justifican en el prefacio de esta obra, que basándose en las investigaciones sobre didáctica de la Física, acerca de las confusiones y dificultades para la apropiación de los conocimientos de ésta, han decantado por utilizar la pedagogía en la mayoría de sus tópicos, sin sacrificar nivel y rigor del texto (Resnick, Halliday y Krane, 2002, p.7). De lo anterior, surge la exhortación para los docentes en activo en ocuparnos de mejorar nuestro quehacer, una opción puede ser empleando aquellas metodologías existentes o de elaboración propia, que proporcionen mejores aprendizajes a nuestros alumnos, así como, indagar en las dificultades que presentan para apropiarse del conocimiento. Es conocida la dificultad - reflejada en el rendimiento académico- y apatía de los estudiantes por las ciencias factuales -como la Física-, aun estudiando ingeniería. Las razones son diversas y multifactoriales. Y, si a lo anterior le sumamos que seguimos instruyendo de la misma forma (clase magistral), evidentemente, los índices de reprobación se mantendrán constantes o al alza. Hasta la fecha no se han instituido metodologías de aprendizaje que mejoren el rendimiento académico en la Academia de Física de la ESIQIE, una alternativa que no impacta de forma exitosa son los talleres de estudio para evaluaciones de título de suficiencia (ETS); desde la percepción de los docentes dichos talleres contribuyen a la preparación de los alumnos para dicha evaluación (aunque dicha percepción no está evidenciada con ninguna investigación). Esta iniciativa viene replicándose en la Academia de Física de la ESIQIE cada semestre, desde el 2015, donde la metodología sigue siendo la clase magistral. No obstante, los resultados no varían, puesto que sólo aprueban en promedio $20 \%$ de los inscritos a esta oportunidad (ETS) de acuerdo al Sistema de Administración Escolar SAES de la ESIQIE (s.f.).

De lo anterior, surge la iniciativa de proponer una alternativa metodológica, Física en el contexto de la Industria Química, que en los sucesivo se denominará FiCtxIQ. Estamos conscientes de que, si seguimos haciendo lo mismo, los resultados no pueden ser distintos. Así, derivado del análisis del perfil de egreso del Ingeniero Químico Industrial (IQI), específicamente de la dimensión saber conocer -como parte del modelo basado en competencias esbozado en el Modelo Educativo del IPN- , se advierte que, de los ocho puntos declarados, $12.5 \%$ se relacionan con el tópico mecánica de fluidos. Es decir, este es un tema que el IQI de la ESIQIE debe dominar, según información de la ESIQIE (s.f): "Los sistemas de conducción de fluidos de procesos químicos y físicos, con base en los principios y modelos de la mecánica de fluidos" (ESIQIE, s.f). Adicionalmente, producto de la indagación realizada por Escobar y Ramírez (2017) la mecánica de fluidos es medular en el entendimiento y aplicación para los ingenieros químicos en ejercicio, puesto que, está ligada a la transferencia de calor y masa; también, al diseño, operación y mantenimiento de plantas químicas. 
Por lo anterior, se decidió que la mencionada alternativa metodológica se centraría en el tópico de dinámica de fluidos.

Es pertinente mencionar, que esta metodología es inédita (hasta antes de la realización de esta investigación, no se encontró otra metodología con características análogas) y en la ESIQIE no se ha aplicado anteriormente. Por lo que, conocer sus alcances y limitaciones es de utilidad para las autoras de esta indagación y eventualmente para docentes de Física a nivel universitario.

Así, el objetivo de esta investigación es determinar si la metodología FiCtxIQ, mejora la comprensión el tópico mecánica de fluidos o no hay mejora significativa; además, de sentar un precedente del tipo de cursos o talleres que se deben impartir para optimizar el rendimiento académico y más relevante es mostrar la relación y relevancia a los estudiantes de lo que se aprende con el futuro ejercicio profesional.

\section{Dificultades en el proceso enseñanza-aprendizaje de la Ecuación de Bernoulli}

La exploración, usanza y creación de metodologías que aseguren la compresión de tópicos importantes, debe ser tarea primordial de todo docente ocupado en cumplir los objetivos del programa de estudios. En este sentido la revisión bibliográfica permite percatarse de la existencia de investigación relacionada con la didáctica de este tópico medular, en la formación de los ingenieros. A continuación se muestra una tabla donde se analizan otras indagaciones sobre el mismo tópico, los alcances, las metodologías y los resultados que obtuvieron en dichas pesquisas (véase tabla 1). 
Tabla 1. Análisis de otras indagaciones relacionadas con dinámica de fluidos.

\begin{tabular}{|c|c|c|c|c|c|}
\hline Autor (es) & $\begin{array}{l}\text { Nivel educativo/ carrera } \\
\text { /tópico }\end{array}$ & Metodología & Resultados & $\begin{array}{l}\text { Semejanzas con } \\
\text { FiCtxIQ }\end{array}$ & $\begin{array}{l}\text { Diferencias } \\
\text { con FiCtxIQ }\end{array}$ \\
\hline $\begin{array}{l}\text { Parra } \\
(2013) .\end{array}$ & $\begin{array}{l}\text { Población universitaria/ } \\
\text { ingeniería/ mecánica de } \\
\text { fluidos. } \\
\text { Se identifica que los } \\
\text { alumnos tienen } \\
\text { conocimientos teóricos de } \\
\text { la mecánica de fluidos; sin } \\
\text { embargo, no saben qué } \\
\text { hacer con ese } \\
\text { conocimiento o su utilidad } \\
\text { para la profesión que } \\
\text { estudian. }\end{array}$ & $\begin{array}{l}\text { Descriptiva, es un } \\
\text { estudio de caso. } \\
\text { Usaron un simulador de } \\
\text { Mecánica de Fluidos } \\
\text { computacional (MFC) } \\
\text { El objetivo fue } \\
\text { desarrollar pensamiento } \\
\text { crítico, mediante } \\
\text { actividades y tareas de } \\
\text { aprendizaje diseñadas } \\
\text { por el profesor y } \\
\text { montadas en la } \\
\text { plataforma Moodle. }\end{array}$ & $\begin{array}{l}\text { Se mide el grado de } \\
\text { satisfacción del aprendizaje } \\
\text { de Mecánica de Fluidos } \\
\text { Computacional mediante } \\
\text { una encuesta. } \\
\text { Donde de acuerdo a los } \\
\text { gráficos presentados los } \\
\text { alumnos aprecian el } \\
\text { simulador, les ayudará para } \\
\text { su vida profesional. }\end{array}$ & $\begin{array}{l}\text { a) Relaciona el } \\
\text { aprendizaje con el } \\
\text { contexto laboral. } \\
\text { b) Realizaron un } \\
\text { instrumento de } \\
\text { elaboración propia } \\
\text { para medir } \\
\text { satisfacción. }\end{array}$ & $\begin{array}{l}\text { La indagación } \\
\text { está basada en } \\
\text { el uso de un } \\
\text { simulador, no } \\
\text { reportan la } \\
\text { efectividad de } \\
\text { la } \\
\text { intervención. }\end{array}$ \\
\hline
\end{tabular}




\begin{tabular}{|c|c|c|c|c|c|}
\hline $\begin{array}{l}\text { Egea, } \\
\text { Pérez y } \\
\text { Franco } \\
(2014) .\end{array}$ & $\begin{array}{l}\text { Población universitaria/ } \\
\text { ingeniería agrícola/ } \\
\text { hidráulica. } \\
\text { Los profesores buscaron } \\
\text { comprobar que se mejoran } \\
\text { las habilidades como } \\
\text { pensamiento, crítico y } \\
\text { creatividad. Además, } \\
\text { proporcionar una } \\
\text { formación integral a los } \\
\text { futuros egresados basada } \\
\text { en competencias. }\end{array}$ & $\begin{array}{l}\text { Cuantitativa, análisis } \\
\text { estadístico inferencial. } \\
\text { Se utilizó la } \\
\text { metodología } \\
\text { Aprendizaje Basado en } \\
\text { Proyectos y Trabajo en } \\
\text { equipo, para incorporar } \\
\text { el enfoque práctico, que } \\
\text { demanda la ingeniería. }\end{array}$ & $\begin{array}{l}\text { No hubo diferencia } \\
\text { significativa con la } \\
\text { metodología explorada. }\end{array}$ & $\begin{array}{l}\text { a) Hicieron un análisis } \\
\text { ANOVA. } \\
\text { b) Percibieron una } \\
\text { actitud favorable } \\
\text { hacia el proceso de } \\
\text { aprendizaje por } \\
\text { parte de los } \\
\text { estudiantes. }\end{array}$ & $\begin{array}{l}\text { a) Utilizaron } \\
\text { un instrumento } \\
\text { de elaboración } \\
\text { propia para } \\
\text { cuantificar la } \\
\text { efectividad de } \\
\text { la } \\
\text { intervención. } \\
\text { b) Su } \\
\text { percepción de } \\
\text { entusiasmo de } \\
\text { los alumnos } \\
\text { está basada en } \\
\text { una creencia. }\end{array}$ \\
\hline $\begin{array}{l}\text { Parra, } \\
\text { Molina, } \\
\text { Luna, } \\
\text { Cacho y } \\
\text { Pérez } \\
(2016) .\end{array}$ & $\begin{array}{l}\text { Población universitaria/ } \\
\text { carreras técnicas. } \\
\text { Buscaron desarrollar el } \\
\text { pensamiento crítico, el } \\
\text { trabajo en equipo, } \\
\text { redacción y evaluación de } \\
\text { informes técnicos. }\end{array}$ & $\begin{array}{l}\text { Descriptiva y } \\
\text { exploratoria, buscan la } \\
\text { opinión sobre la } \\
\text { metodología empleada. } \\
\text { La metodología } \\
\text { propuesta pretendió } \\
\text { elevar la comprensión } \\
\text { de tópicos de Mecánica } \\
\text { de Fluidos a través de } \\
\text { videos cortos utilizando } \\
\text { códigos QR. }\end{array}$ & $\begin{array}{l}\text { Se muestra con la } \\
\text { estrategia, una mejoría en } \\
\text { la percepción de los } \\
\text { tópicos. }\end{array}$ & $\begin{array}{l}\text { Los autores registraron } \\
\text { una percepción } \\
\text { favorable al método } \\
\text { por parte de los } \\
\text { estudiantes. }\end{array}$ & $\begin{array}{l}\text { a) Utilizaron } \\
\text { una encuesta } \\
\text { de satisfacción } \\
\text { de elaboración } \\
\text { propia. } \\
\text { Hacen uso de } \\
\text { dispositivos } \\
\text { móviles (al } \\
\text { igual que en } \\
\text { nuestra } \\
\text { indagación) } \\
\text { para acceder } \\
\text { con códigos } \\
\text { QR a videos } \\
\text { ilustrativos (en } \\
\text { nuestro caso }\end{array}$ \\
\hline
\end{tabular}




\begin{tabular}{|c|c|c|c|c|c|}
\hline & & & & & $\begin{array}{l}\text { para acceder a } \\
\text { Kahoot!) }\end{array}$ \\
\hline $\begin{array}{l}\text { Álvarez, } \\
\text { Castillo, } \\
\text { Pizarro y } \\
\text { Espinoza } \\
(2017) .\end{array}$ & $\begin{array}{l}\text { Población universitaria/ } \\
\text { ingeniería industrial. } \\
\text { Buscaron desarrollar una } \\
\text { experiencia de realidad } \\
\text { aumentada para el proceso } \\
\text { de aprendizaje de la } \\
\text { mecánica de fluidos. }\end{array}$ & $\begin{array}{l}\text { Descriptiva y } \\
\text { exploratoria, buscaron } \\
\text { la opinión sobre la } \\
\text { metodología empleada. } \\
\text { Seleccionaron el caso, } \\
\text { el hardware y software } \\
\text { para generar los } \\
\text { elementos de realidad } \\
\text { aumentada, su código } \\
\text { AR (Realidad } \\
\text { Aumentada), probaron } \\
\text { y validaron el Objeto } \\
\text { Virtual de Aprendizaje. }\end{array}$ & $\begin{array}{l}\text { La realidad aumentada } \\
\text { permitió que los } \\
\text { estudiantes } \\
\text { pudieran visualizar } \\
\text { íntegramente los } \\
\text { problemas. }\end{array}$ & $\begin{array}{l}\text { Utilizaron la encuesta } \\
\text { de opinión calibrada y } \\
\text { validad (Jaramillo), } \\
\text { propuesta por otro } \\
\text { autor para valorar el } \\
\text { impacto de las } \\
\text { estrategias a través de } \\
\text { enseñanzas visuales. }\end{array}$ & $\begin{array}{l}\text { Registraron } \\
\text { por parte de } \\
\text { los estudiantes, } \\
\text { una percepción } \\
\text { y aceptación } \\
\text { favorable al } \\
\text { método } \\
\text { propuesto. }\end{array}$ \\
\hline $\begin{array}{l}\text { Fernández, } \\
\text { Fernández } \\
\text { Oro, } \\
\text { Menéndez, } \\
\text { Galdo, } \\
\text { Barrio y } \\
\text { Blanco } \\
(2018) .\end{array}$ & $\begin{array}{l}\text { Población universitaria/ } \\
\text { ingeniería/ mecánica de } \\
\text { fluidos. } \\
\text { Los profesores reportan } \\
\text { índices de reprobación de } \\
\text { entre } 30 \text { y } 40 \% \text { y perciben } \\
\text { a los estudiantes } \\
\text { desmotivados. }\end{array}$ & $\begin{array}{l}\text { Exploratoria, ya que, se } \\
\text { buscan probar otras } \\
\text { estrategias. Así, } \\
\text { utilizaron la } \\
\text { gamificación, como } \\
\text { complemento para } \\
\text { reforzar el } \\
\text { conocimiento } \\
\text { adquirido. }\end{array}$ & $\begin{array}{l}\text { Se mostró que con esta } \\
\text { estrategia se pueden } \\
\text { derivar otras líneas de } \\
\text { actuación para mejorar la } \\
\text { calidad académica. }\end{array}$ & $\begin{array}{l}\text { Se utiliza la } \\
\text { gamificación con } \\
\text { doble propósito, para } \\
\text { activar conocimientos } \\
\text { previos y para } \\
\text { retención de conceptos } \\
\text { clave. }\end{array}$ & $\begin{array}{l}\text { Nuestra } \\
\text { propuesta } \\
\text {, no está } \\
\text { basada sólo en } \\
\text { gamificación, } \\
\text { es una parte de } \\
\text { todo el } \\
\text { proceso. }\end{array}$ \\
\hline
\end{tabular}




\section{Metodología}

La indagación es de tipo experimental, donde la variable independiente es la metodología de enseñanza-aprendizaje y la variable dependiente es el nivel de entendimiento sobre el tema dinámica de fluidos (notas obtenidas por cada alumno de cada grupo). También es descriptiva ; porque, se analizan e interpretan los hallazgos.

La población estuvo constituida por 74 alumnos de la licenciatura de Ingeniería Química Industrial de la ESIQIE, de los cuales el 45\% fueron de género femenino y el 55\% de género masculino, con un rango de edades de 18 a 29 años de edad. De esta población el 31\% eran irregulares (no han aprobado la unidad de aprendizaje mecánica clásica). El 14\% manifestó que trabaja.

Este experimento se efectuó con tres grupos, donde el proceso de selección de los tres grupos fue aleatorio simple. Y se llevó a cabo en las instalaciones de la ESIQIE, en cada grupo se implementó una metodología de enseñanza-aprendizaje diferente y con tiempos diferentes, una respecto de la otra (véase tabla 2).

Tabla 2. Descripción de los grupos a los que se realizó la indagación.

\begin{tabular}{ccc} 
Grupo & Metodología & Tiempo \\
1 & Repetición-recepción & 4 horas (240 minutos) \\
2 & Física recreativa + & 7 horas (420 minutos) \\
& $\begin{array}{c}\text { ejercicios + Problema de } \\
\text { la Industria Química }\end{array}$ & \\
3 & FiCtxIQ & 12 horas (720 minutos) \\
\hline
\end{tabular}

Acerca del proceso de recolección de datos, se efectúo de forma escrita (hoja de respuestas), en los momentos pre-test y post-test. Es pertinente señalar que los datos arrojados por el post-test son los que se utilizaron para la realización del análisis de la varianza unifactorial (ANOVA) para los tres grupos. Tal como, señala Devore $(2009$, p.369) es útil para la investigación de datos muestreados de más de dos grupos, en los cuales se utilizaron más de dos métodos. Además, los datos pre-test y post-test, resultaron valiosos para valorar si hay mejora del conocimiento de este tópico específico con la metodología FiCtxIQ mediante el factor de Hake (1998), esto último se analizó sólo para el grupo dos.

La presente indagación se realizó en las siguientes etapas:

Etapa 1. Se analizaron los índices de reprobación de la unidad de aprendizaje mecánica clásica. Y se discutió, sobre la necesidad de realizar acciones que mejoraran el rendimiento académico, sin sacrificar contenidos ni rigor académico. Porque, lo anterior se concibió como una problemática que debe; además de abordarse, resolverse.

Etapa 2. Se indagó sobre las dificultades de aprendizaje (con los alumnos, entrevistas personalizadas) y análisis de sus evaluaciones; sobre los tópicos relevantes del contenido programático de mecánica clásica en el 
ejercicio profesional, esto se realizó con ingenieros egresados, mediante una indagación basada en encuestas y entrevistas realizadas y reportadas por Escobar y Ramírez (2017). Se encontró, de acuerdo a lo que reportan los mencionados autores, que la relevancia del tópico dinámica de fluidos en la formación de los ingenieros, es medular para la operación de una planta o fábrica; además, de su estrecha vinculación con la transferencia de calor y transferencia de masa.

Etapa 3. Se consideraron los índices de reprobación y la indagación realizada por Escobar y Ramírez (2017) y se dispuso a evaluar el impacto que tendría la instrucción de dicho tópico con tres metodologías diferentes.

Etapa 4. Se aplicó el test de LPHB, Ley de Presión Hidrodinámica de Bernoulli de Barbosa (2013), el cual proporcionó el nivel de conocimientos de los alumnos de cada grupo.

Etapa 5. Se implementaron tres metodologías de enseñanzaaprendizaje diferentes con cada uno de los grupos. Es pertinente mencionar que con los tres grupos interactuó la misma profesora. A continuación, se describe la metodología con cada grupo.

Grupo uno. Enseñanza exposición- recepción (clase magistral). La profesora resuelve cuatro ejercicios que se proponen en la bibliografía, resuelve dudas y las actividades de aprendizaje son: cinco ejercicios con respuestas.

Grupo dos. Física recreativa + resolución de ejercicios+ problema de la Industria Química. La profesora presenta 4 experimentos relacionados con dinámica de fluidos, apoyado en guías de discusión (véase apéndice B); después, se procede a la aplicación de cinco ejercicios relacionados con dinámica de fluidos, pero, con la variante de que los alumnos los resuelven en equipo, de estos ejercicios, uno explica la profesora al detalle y otro ejercicio se les presenta el algoritmo y los alumnos deben redactar un ejercicio que satisfaga ese algoritmo, se les proporciona la realimentación sobre la solución de los ejercicios. Finalmente se procede a la presentación de un problema de la Industria Química, dónde se les solicita planteen la solución en el marco de la Física. La solución de este problema se apoya en cinco pasos, que incluye: lectura y comprensión, indagación, formulación de hipótesis, diagrama de espina de pescado, soluciones y recomendaciones. Esta última parte la hacen fuera del aula.

Grupo tres. Metodología Física en el contexto de la Industria Química (FiCtxIQ). La cual incluye: gamificación + Física recreativa + resolución de ejercicios+ Problema de la Industria Química. Primeramente la profesora presenta el encuadre teórico mediante un juego en línea (véase apéndice C) creado en la herramienta gratuita y digital Kahoot! https://create.kahoot.it/details/conceptos-importantes-de-hidraulica/ffb3efced4f8-4d86-a5d3-80f64bd87eec 
Esta herramienta se utilizó para revisar los conceptos mínimos necesarios que se asocian a la dinámica de fluidos, porque, las estrategias que se sugieren como evidencias de aprendizaje se limitan a realizar investigaciones o ensayos. De dichas evidencias no existe la seguridad de que las realice el alumno, puede copiarlas o realizarlas sin retener la información. La gamificación se utilizó como herramienta para activar conocimientos; además, para conocer el nivel de conocimientos previos de los alumnos. Si bien, no hubo un puntaje asignado para los alumnos puesto que la metodología se estuvo probando en el taller para medir su efectividad, si se les percibió entusiastas e interesados. No obstante, a los equipos ganadores (3 mejores puntajes) la plataforma Kahoot! al final los muestra en un podio con medallas (los equipos ganadores se notaron jubilosos por tal logro).

Posteriormente, presenta 5 experimentos relacionados con dinámica de fluidos, apoyado en guías de discusión. Uno de los experimentos debe ser propuesto por los alumnos, el material a utilizar es una jeringa; después, se procede a la aplicación de cinco ejercicios relacionados con dinámica de fluidos, pero con la variante de que los alumnos los resuelven en equipo. De estos ejercicios, uno lo explica el profesor al detalle y del otro ejercicio se les presenta el algoritmo y los alumnos deben redactar un ejercicio que satisfaga ese algoritmo; se les proporciona la realimentación. Finalmente se procede a la presentación de un problema de la Industria Química, dónde se les solicita planteen la solución en el marco de la Física. La solución de este problema se apoya en cinco pasos, que incluye: lectura y comprensión, indagación, formulación de hipótesis, diagrama de espina de pescado y soluciones y recomendaciones. Esta última parte la hacen en el aula.

Esta metodología es parte de un proyecto de investigación que se está afinando y robusteciendo en la ESIQIE y en el Centro de Investigación de Ciencia Aplicada y Tecnología Avanzada (CICATA) del Instituto Politécnico Nacional (IPN). Se parte de la premisa que los alumnos deben ser edificadores de sus saberes, no obstante, estos deben ser parte de un proceso de andamiaje cognitivo. El andamiaje cognitivo de acuerdo con Wood, Bruner y Ross citado por Zazueta, M. \& Herrera, L. (2009), se concibe como un esqueleto provisional; como parte de una táctica cuyo fin es apoyar a los estudiantes a estructurar la información en segmentos relevantes, examinarlos y elaborar soluciones nuevas, habilidades y conceptos propios. Con la estructura propuesta (4 pasos) para FiCtxIQ se considera un andamiaje cognitivo, necesario para lograr resolver el problema de la Industria Química relacionado con dinámica de fluidos.

En resumen, esta metodología está compuesta de 4 pasos: gamificación, Física recreativa, ejercicios y problema de la Industria Química.

Las notas se obtuvieron como ya se mencionó, mediante la aplicación del instrumento calibrado y validado denominado Test (LPHB) de la Ley de 
Presión Hidrodinámica de Barbosa de Barbosa (2013). Se analizaron con la función complementos para analizar datos de Microsoft Excel 2013, aplicando la función "Análisis de varianza de un factor" con un 95\% de confianza.

Adicionalmente, se aplicó un instrumento de elaboración propia para indagar sobre la satisfacción del taller donde se aplicó FiCtxIQ larga (el instrumento sólo se aplicó con el grupo dos). Partiendo de la premisa que el proceso enseñanza aprendizaje desde el punto de vista de calidad, es un servicio educativo y en concordancia con la Actualización del Plan de Desarrollo Institucional 2015-2018 del Instituto Politécnico Nacional, que en el Proyecto Especial 02, pretende: "Establecer el Sistema Institucional de Evaluación de la Calidad Educativa[...]" (PDI 2015-2018, p.34). Entonces, se considera que es pertinente promover una cultura de la calidad en la docencia (enfocado a la calidad en el servicio), por lo que, monitorear la percepción de éstos servicios por parte de los alumnos que recibieron dicho taller; también, es relevante en esta pesquisa.

El instrumento de elaboración propia (véase apéndice A) para cuantificar la satisfacción de los alumnos respecto al taller donde se aplicó la metodología FiCtxIQ se basó en lo que propone Evans y Lindsay (2010, p.166) sobre las dimensiones que debe contemplar un servicio, en nuestro caso la adaptación se realizó para un servicio educativo, como anteriormente se menciona. Las dimensiones evaluadas fueron:

1. Confiabilidad: la capacidad de proveer lo que se prometió, con seguridad y exactitud.

2. Aseguramiento: el conocimiento y cortesía de los empleados (profesores), así como su capacidad para transmitir confianza.

3. Tangibles: las instalaciones y equipo, así como la apariencia del personal (profesores).

4. Empatía: grado de cuidado y atención individual que se ofrece a los clientes (alumnos).

5. Capacidad de respuesta: la disposición para ayudar a los clientes (alumnos) y prestarles un servicio oportuno.

\section{Resultados}

Se presenta un resumen de los datos estadísticos obtenidos por los tres grupos (véase tabla 3) y los datos estadísticos en los momentos pre-test y posttest del grupo dos (véase tabla 4).

Tabla 3. Datos estadísticos de los tres grupos (post-test).

\begin{tabular}{|c|c|c|c|}
\hline \multirow{2}{*}{ Valores estadísticos } & \multicolumn{3}{|c|}{ Post-test } \\
\cline { 2 - 4 } & Grupo 1 & Grupo 2 & Grupo 3 \\
\hline Número de participantes & 20 & 17 & 34 \\
\hline Promedio & 2.25 & 4.21 & 4.58 \\
\hline Mediana & 2.08 & 5.00 & 4.58 \\
\hline
\end{tabular}




\begin{tabular}{|c|c|c|c|}
\hline Moda & 1.67 & 5.83 & 5.00 \\
\hline Desviación estándar & 1.29 & 2.12 & 1.93 \\
\hline Varianza & 0.18 & 0.47 & 0.39 \\
\hline Máximo & 5 & 7 & 9 \\
\hline Mínimo & 0 & 0 & 1 \\
\hline
\end{tabular}

Tabla 4. Datos estadísticos del grupo dos (pre-test y post-test).

\begin{tabular}{|c|c|c|}
\hline Valores estadísticos & Pre-test & Post-test \\
\hline Número de participantes & 17.00 & 17.00 \\
\hline Promedio & 2.25 & 4.21 \\
\hline Mediana & 2.08 & 5.00 \\
\hline Moda & 1.67 & 5.83 \\
\hline Desviación estándar & 1.43 & 2.12 \\
\hline Varianza & 0.18 & 0.47 \\
\hline Máximo & 5 & 7 \\
\hline Mínimo & 0 & 0 \\
\hline
\end{tabular}

Previo al uso de la prueba paramétrica ANOVA se confirmó la distribución normal de los datos de cada grupo, a través de los gráficos QQ plots. Así, se observa en la gráfica que los puntos siguen una trayectoria rectilínea $\left(\mathrm{a} 45^{\circ}\right)$ a la línea de tendencia (véase figura 1$)$. Se muestra el gráfico correspondiente al grupo dos.

Figura 1. Gráfico QQ plot para grupo dos

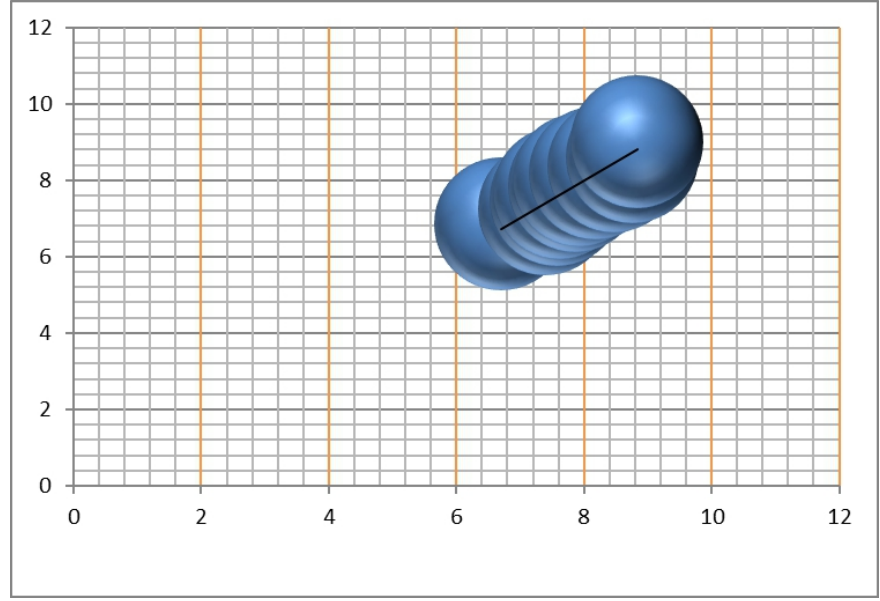

Así, mediante un análisis de varianza unidireccional se propone que:

H0: $\mu 1=\mu 2=\mu 3 \leftarrow$ Hipótesis nula

$\mathrm{H} 1: \mu 1, \mu$ y $\mu 3$ no son todas iguales $\leftarrow$ Hipótesis alternativa

Tal como, se mencionó se empleó un ANOVA unifactorial para aceptar o rechazar la hipótesis. Se muestran los resultados del ANOVA (véase tabla 5): 
Tabla 5. Tabla ANOVA para los tres grupos.

\begin{tabular}{|c|c|c|c|c|c|c|}
\hline $\begin{array}{l}\text { Origen de } \\
\text { las } \\
\text { variaciones }\end{array}$ & $\begin{array}{l}\text { Suma de } \\
\text { cuadrados }\end{array}$ & $\begin{array}{l}\text { Grados } \\
\text { de } \\
\text { libertad }\end{array}$ & $\begin{array}{l}\text { Promedio de } \\
\text { los cuadrados }\end{array}$ & $\mathbf{F}$ & Probabilidad & $\begin{array}{c}\text { Valor crítico } \\
\text { para } F\end{array}$ \\
\hline $\begin{array}{l}\text { Entre } \\
\text { grupos }\end{array}$ & 0.720523649 & 2 & 0.360261824 & 10.1847557 & 0.0001292 & 3.125764237 \\
\hline $\begin{array}{l}\text { Dentro de } \\
\text { los grupos }\end{array}$ & 2.511458333 & 71 & 0.035372653 & & & \\
\hline Total & 3.231981982 & 73 & & & & \\
\hline
\end{tabular}

Se logra constatar que la columna $F$ arroja un valor de 10.1847557 y la columna Valor crítico para $F$ arroja el valor de 3.125764237 por lo que se rechaza la hipótesis nula.

Esto indica, al nivel de significancia de 0.05 , que hay evidencia estadística que señalan que sí existen diferencias entre las distintas metodologías de instrucción y sus efectos se perciben en las notas derivadas del Test LPHB. De manera análoga se precisa en la columna Probabilidad, que arroja un valor 0.0001292 , el cual es menor al nivel de significancia $(\alpha=0.05)$.

Del grupo dos se analizó la mejora del conocimiento respecto al tópico dinámica de fluidos, obtenida producto de la metodología FiCtxIQ con duración de 12 horas. Así la métrica denominada factor de Hake, cuantificó el aprendizaje de la metodología FiCtxIQ en función de la fórmula que permite el cálculo de este índice, de acuerdo con Hake (1998):

$$
g=\frac{\text { postest } \%-\text { pretest } \%}{100-\text { pretest } \%}
$$

Este índice establece que la metodología FiCtxIQ está en el rango de una ganancia media, ya que, es mayor a 0.3 (véase tabla 6) señala Hake (1998).

Tabla 6. Factor de Hake del grupo dos

\begin{tabular}{cccc}
\hline Grupo & Pre-test (\%) & Post-test(\%) & Factor de Hake \\
2 & $22.50 \%$ & $42.10 \%$ & 0.34 \\
\hline
\end{tabular}

Se muestra del grupo dos, los resultados de satisfacción (véase figura 2) obtenidos del instrumento de elaboración propia basado en las cinco dimensiones de calidad en el servicio previamente descritas, producto del Taller donde se implementó la metodología FiCtxIQ. Los gráficos revelan que la instructora obtuvo el $85.82 \%$ y $14.18 \%$ de satisfacción total y general, respectivamente. Algo resaltable a mencionar es la atención que recibió el grupo dos, al cual adicionalmente se le pagaron viáticos (la inversión de 40 USD, cubierta por las autoras de este trabajo) para alentar la asistencia al taller que se impartió en el periodo intersemestral 2019/1 -periodo vacacional para los alumnos-. Además, se les informó sobre los objetivos del taller, de la forma 
de evaluar, se les retroalimentó en tiempo y forma, se respetaron los acuerdos de evaluación, se les respondieron sus dudas de forma afable, se les tutelaba por su nombre.

Figura 2. Resultados de satisfacción del grupo dos.

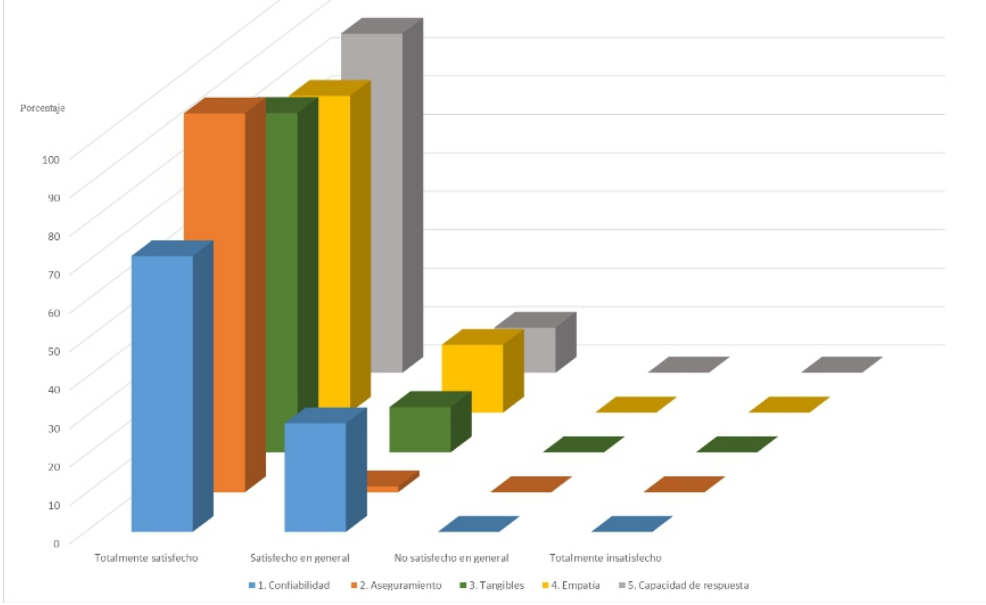

\section{Discusión}

Se observa que existe una mejora con las metodologías asociadas al grupo dos y tres. Se considera que tenemos inferencias válidas respecto a la certidumbre de la utilidad de las metodologías utilizadas. Es resaltable que el grupo dos estuvo dedicado exclusivamente a atender las asignaciones del taller y se tuvo especial espero en los servicios educativos. Del mismo modo, llama la atención que su rendimiento fue ligeramente inferior al del grupo tres, es posible que haya influido el hecho que para el grupo tres la realización de la metodología era parte de su evaluación continua y por tal razón estaban prestos. Además, de doblar en número de participantes al grupo dos. También, se debe resaltar que los productos de aprendizaje del grupo tres obtuvieron notas más altas, pese a que no hicieron la actividad en el aula. El grupo dos estuvo supervisado por la instructora. El grupo dos es un grupo donde los alumnos no han aprobado la unidad de aprendizaje mecánica clásica, es decir, de acuerdo a sus calificaciones y promedios globales presentan bajo rendimiento académico. Pese a lo anterior, la evidencia estadística muestra que hay mejora significativa con FiCtxIQ. Respecto a las otras investigaciones (al menos las consultadas) utilizando metodologías de aprendizaje activo o nuevas tendencias en la instrucción de la Física, en específico para el tópico dinámica de fluidos; encontramos que sólo una pretendió medir a través de un estadístico de prueba su efectividad, corroborando que no hubo mejora en el aprendizaje. Las otras indagaciones se basaron en explorar o describir la experiencia del uso de metodologías de aprendizaje (Aprendizaje Basado en 
Proyectos, uso de códigos AR, etc). De las indagaciones revisadas ninguna es inédita, por lo que, consideramos que nuestra aportación es relevante puesto que estamos proponiendo una metodología pertinente para nivel universitario y que integra elementos que propician la motivación, trabajo en equipo, creatividad y resolución de problemas complejos. Resaltando que las dos últimas son las habilidades que demanda la Cuarta Revolución Industrial, señala Moy (2016).

\section{Conclusión}

Pese a que se revisó la misma temática con los tres grupos, hay evidencia estadística que señala que las metodologías utilizada si impactan de forma tangible. Producto de esta indagación, se evidencia la necesidad de migrar a otras formas de instruir; también, se resalta el insertar desde la labor docente la calidad en el servicio. Es conveniente que la metodología FiCtxIQ se replique de nuevo; pero con un grupo más avanzado, puesto que el Problema de la Industria Química que se les presentó les resultó complejo. Entre otras cosas; porque el $98 \%$ de los estudiantes no se les había planteado un problema abierto con esas características; presentaron dificultades para categorizar el problema en el marco de la Física (específicamente dinámica de fluidos). Ya que, lo usual es resolver ejercicios donde deben despejar fórmulas y se invita a hallar una o más incógnitas. La sugerencia derivada de los datos obtenidos, es replantear la docencia. Porque, además de que se incrementa notoriamente el aprendizaje, se perciben alumnos interesados, motivados, pensantes y muy participativos. Asimismo, se les adosa a su futura realidad laboral, que es incierta y que se vaticina tendrá retos más complejos.

No se puede soslayar mencionar que la preparación de toda la metodología requirió más de 16 horas de trabajo previo, es decir, por mucho es menos trabajo que si se preparara una clase de forma tradicional.

\section{References:}

1. Alvarez A., Castillo M., Pizarro J., y Espinoza E. (2017). Realidad Aumentada como Apoyo a la Formación de Ingenieros Industriales. Formación universitaria, 10(2), 31-42.

2. Barbosa, L. H. (2013). Construcción, validación y calibración de un instrumento de medida del aprendizaje: Test de Ley de Bernoulli. Revista Educación en Ingeniería, 8(15), 24-37.

3. Devore, J. L. (2009). Probabilidad y estadística para ingeniería y ciencias. Cengage Learning Editores.

4. Escobar, F. y Ramírez, M. (2017). Eventos contextualizados de la industria química para el aprendizaje del Principio de Bernoulli. Presentado en el XXV Taller Internacional: Nuevas Tendencias en la Enseñanza de la Física, Puebla, Pue. 
5. Egea G., Pérez L. y Franco A. (2014). Aprendizaje basado en proyectos y trabajo en equipo: experiencia de innovación docente en hidráulica para graduados en Ingenierá Agrícola. Universidad de Sevilla. AGR268: Naturación Urbana e Ingeniería de Biosistemas. Recuperado de: http://dspace.sheol.uniovi.es/dspace/bitstream/10651/48173/1/CUIEE TMeana.pdf

6. ESIQIE (s.f.). [Fecha de consulta: 9 de enero del 2019]. Disponible en:

7. https://www.esiqie.ipn.mx/oferta-educativa/vercarrera.html? $\mathrm{g}=\mathrm{es} \& \mathrm{id}=20$

8. Evans, J., y Lindsay, W. (2010). Administración y control de la calidad. Cengage Learning Editores.

9. Fernández, Fernández Oro, Menéndez, Galdo, Barrio y Blanco (2018). Tendencias en la innovación docente en enseñanzas técnicas: análisis y propuesta de mejoras para la asignatura Mecánica de Fluidos. libro de actas deL XXVI Congreso Universitario de Innovación Educativa En las Enseñanzas Técnicas (pp. 349-360). España: Escuela Politécnica de Ingeniería de Gijón Universidad de Oviedo.

10. Hake, R (1998). Interactive engagement vs traditional methods: A sixthousand student survey of mechanics test data for introductory physics course. Am. J. Phys. 66, 64-74.

11. Instituto Politécnico Nacional (2018). Actualización Programa de Desarrollo Institucional 2015-2018. México: IPN. Recuperado de: http://www.gestionestrategica.ipn.mx/Planeacion/Documents/ActPDI 1518.pdf

12. Kahoot!.com. Generador de juegos. [Online] Disponible en: <https://kahoot.com/> [Accesado el 26 de enero del 2018].

13. Moy, V. (20 de enero del 2016). Davos y la Cuarta Revolución Industrial. El Financiero. Recuperado de: http://www.elfinanciero.com.mx/opinion/davos-y-la-cuartarevolucion-industrial.html

14. Parra, T. (2013). Aprendizaje Práctico de Mecánica de Fluidos Computacional usando TIC. En V Congreso Internacional Latina de Comunicación Social.

15. Parra, M., Molina, J., Luna G., Cacho M., Pérez J. (2016). Integración de Códigos de Respuesta Rápida para Agilizar el Desarrollo de la Clase de Aula. (PID 1516). Recuperado de: https://uvadoc.uva.es/bitstream/10324/18751/1/ANEXOIVPID_1516 _068.pdf

16. Resnick, R., Halliday, D. y Krane, K. (2002) Física Vol. I. Patria. 
17. Sistema de Administración Escolar de la ESIQIE (s.f.) .). [Fecha de consulta: 4 de febrero del 2019]. Disponible en: https://www.saes.esiqie.ipn.mx/

18. Zazueta, M. y Herrera, L. (2009). Andamio cognitivo herramienta para el proceso de aprendizaje. Quaderns digitals: Revista de Nuevas Tecnologías y Sociedad, (60), 12.

\section{Apéndice A. Instrumento de satisfacción del Taller Mecánica de fluidos}

Estimado alumno, te solicitamos respondas las siguientes interrogantes de forma honesta y responsable. La finalidad es encontrar áreas de mejora y así contribuir a tu formación profesional integral.

Nombre del Taller:

Academia: Lugar:

Nombre del instructor: Fecha:

\begin{tabular}{|c|c|}
\hline \multicolumn{2}{|c|}{ Escala de puntuación } \\
\hline 4 & Totalmente satisfecho \\
\hline 3 & Satisfecho en general \\
\hline 2 & No satisfecho en general \\
\hline 1 & Totalmente insatisfecho \\
\hline
\end{tabular}

\begin{tabular}{|c|c|c|c|c|c|}
\hline $\mathrm{P}$ & Nivel de satisfacción & 4 & 3 & 1 & 0 \\
\hline 1 & $\begin{array}{l}\text { Usa una metodología de enseñanza participativa, activa y } \\
\text { productiva }\end{array}$ & & & & \\
\hline 2 & Muestra dominio de los temas que se abordan & & & & \\
\hline 3 & Explica el lenguaje técnico utilizando palabras que conozcas & & & & \\
\hline 4 & Utiliza métodos de enseñanza atractivos & & & & \\
\hline 5 & $\begin{array}{l}\text { Contextualiza sus clases y las vincula con situaciones de la } \\
\text { realidad }\end{array}$ & & & & \\
\hline 6 & Se expresa de forma apropiada y clara & & & & \\
\hline 7 & Responde tus dudas de forma correcta & & & & \\
\hline 8 & $\begin{array}{l}\text { Brinda orientaciones para trabajo independiente y uso de } \\
\text { material de estudio por diversas vías }\end{array}$ & & & & \\
\hline 9 & Se muestra dispuesto a atender tus dudas & & & & \\
\hline 10 & Te da la confianza para expresar tus dudas & & & & \\
\hline 11 & $\begin{array}{l}\text { Atiende tus solicitudes (aclaraciones, explicaciones, } \\
\text { retroalimentaciones) de forma rápida }\end{array}$ & & & & \\
\hline 12 & Es amable en su trato & & & & \\
\hline 13 & Se dirige a ti por tu nombre & & & & \\
\hline 14 & $\begin{array}{c}\text { Atiende las diferencias de aprendizaje y diversidad de los } \\
\text { alumnos }\end{array}$ & & & & \\
\hline 15 & $\begin{array}{l}\text { Estimula y reconoce los resultados de los estudiantes de } \\
\text { acuerdo con sus diferentes posibilidades }\end{array}$ & & & & \\
\hline 16 & Retroalimentó tu tarea en tiempo y forma & & & & \\
\hline
\end{tabular}




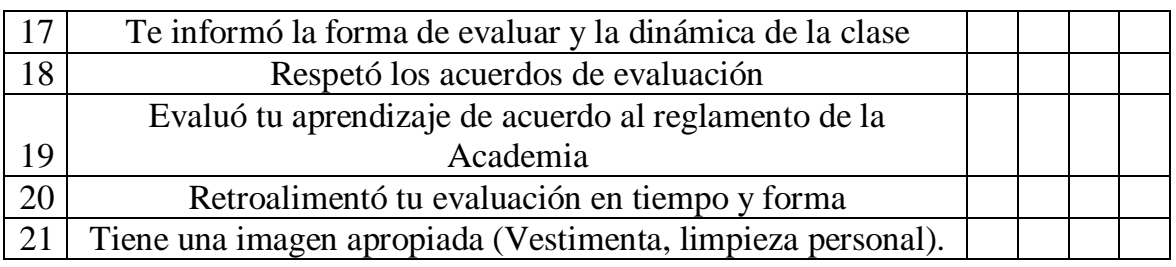

\section{Apéndice B. Guías de discusión para física recreativa}

\begin{tabular}{|c|c|}
\hline $\begin{array}{l}\text { La hoja pesada } \\
\text { En esta experimentación comprenderás la } \\
\text { afectación de la presión atmosférica en los } \\
\text { objetos. }\end{array}$ & $\begin{array}{l}\text { Instrucciones: } \\
\text { 1. Coloca una regla sobre una mesa (véase } \\
\text { figura 1) y encima de la regla, una hoja } \\
\text { completa de periódico. } \\
\text { 2. Golpea el extremo saliente de la regla y } \\
\text { observa. } \\
\text { 3. Tome la misma hoja de periódico y } \\
\text { estrújela, colócala después en la regla, } \\
\text { golpea el extremo saliente de la regla y } \\
\text { observa. }\end{array}$ \\
\hline Materiales & \\
\hline $\begin{array}{l}\text { - } 2 \text { hojas } \\
\text { - Regla }\end{array}$ & ra 1. Experimento realizado por los alumnos \\
\hline \multicolumn{2}{|c|}{$\begin{array}{l}\text { Preguntas } \\
\text { a) ¿Pudiste rotar la hoja de periódico completa? } \\
\text { b) ¿Qué ocurrió con la hoja de periódico estrujada (bola)? } \\
\text { c) ¿A qué se debe este fenómeno? }\end{array}$} \\
\hline
\end{tabular}


Conclusiones

\section{El embudo agorero}

En esta experimentación comprenderás la paradoja de fluidos, cuando en un embudo invertido se sustenta una pelota por el flujo de aire que tiene dirección descendente.

\section{Instrucciones:}

1. Coloca la pelota dentro de un embudo (véase figura 2).

2. Sopla fuerte y rápido.

3. Sin dejar de soplar voltea el embudo con la pelota dentro y observa.
- Embudo de vidrio

- Pelota de unicel

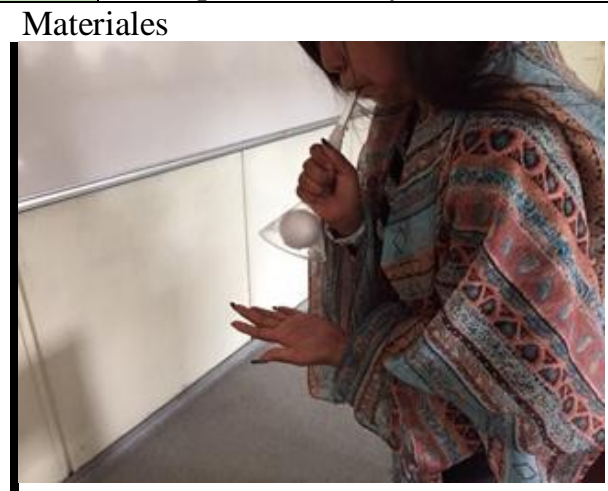

Figura 2. Experimento realizado por los alumnos

Preguntas

a) Mientras soplabas, ¿qué ocurrió con la pelota de unicel?

b) ¿La velocidad del aire encima de la pelota aumenta o disminuye con el soplido?

c) ¿De qué forma el principio de Bernoulli explica el comportamiento de la moneda durante el soplido?

d) De acuerdo con el principio de Bernoulli, al aumentar la velocidad del aire encima de la pelota, ¿qué ocurre con la presión encima de ésta?

Conclusiones

\section{Botella porosa}

Con este experimento aprenderás que gasto de un fluido representa la cantidad del fluido que se desplaza en un intervalo de tiempo.

\section{Instrucciones:}

1. Realiza perforaciones pequeñas a lo largo de una línea vertical en un costado del recipiente y enuméralas (véase figura 3 ).

2. Tapa los orificios con cinta y después agregue agua hasta llenarlo.

3. Destapa los orificios. 


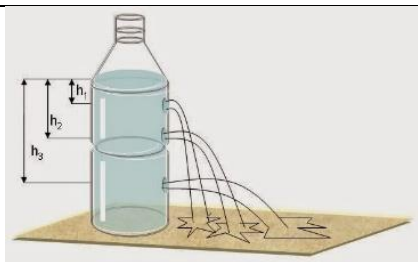

- Botella de plástico $500 \mathrm{ml}$

Figura 3. Modelo del experimento que realizaron los alumnos

- Agua

- Regla

- Aguja gruesa

Preguntas

a) El agua, ¿tiene el mismo alcance?

b) Todos los chorros de agua, ¿tienen la misma velocidad?

Conclusiones

\section{Latas repulsivas}

Con este experimento observarás que la presión del aire entre las latas es menor y origina que las latas se empujen una a la otra.

\section{Instrucciones:}

1. Ubica cada lata sobre dos de los popotes, con un espacio entre ellas de $1 \mathrm{~cm}$ (véase figura 4).

2. Con el otro popote sopla a través de la separación entre las latas.

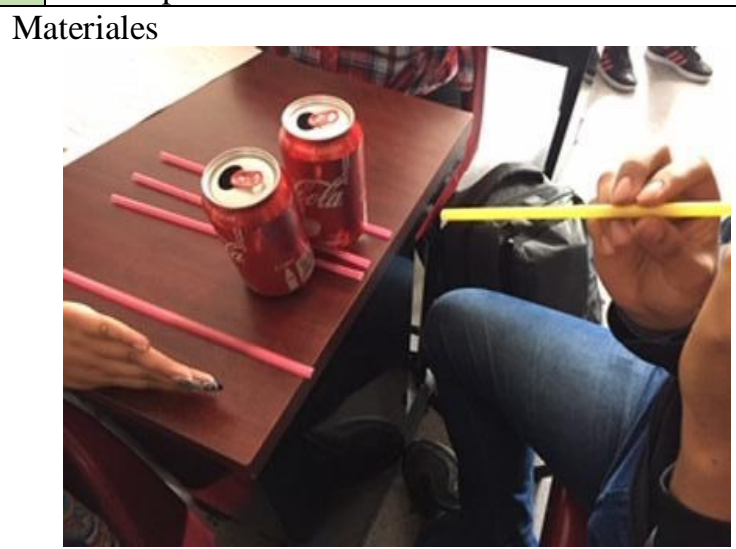

Figura 4. Experimento realizado por los alumnos

- 2 latas de refresco vacías

- 5 popotes

Preguntas

a) Antes de soplar, ¿qué esperas que suceda?

b) ¿Qué sucede con las latas?

c) ¿Qué principio explica este resultado?

Conclusiones 


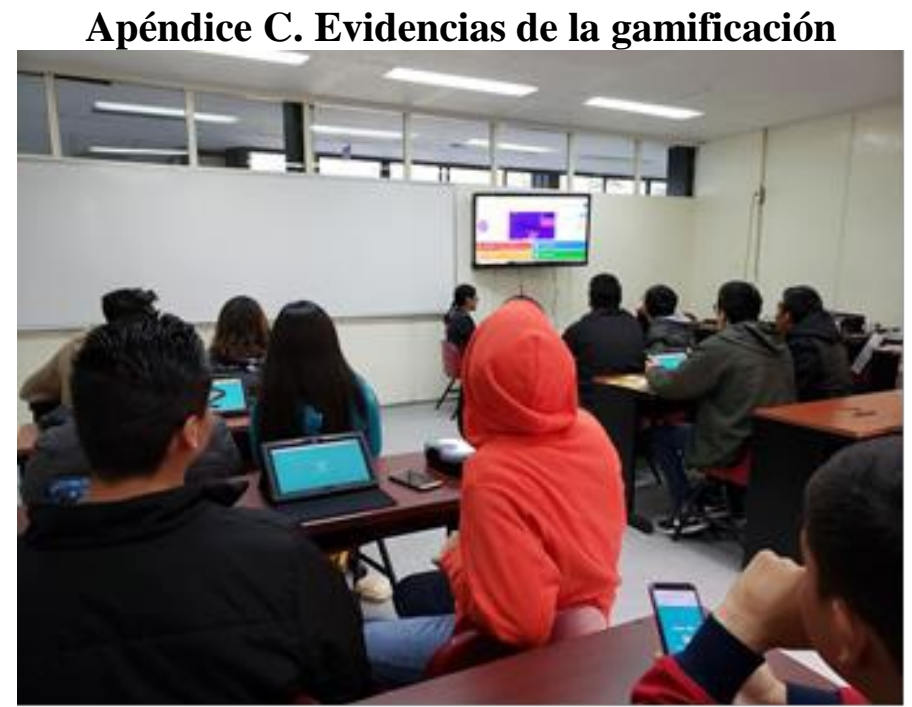

Figura 5. Alumnos gamificando, con tabletas y teléfonos móviles

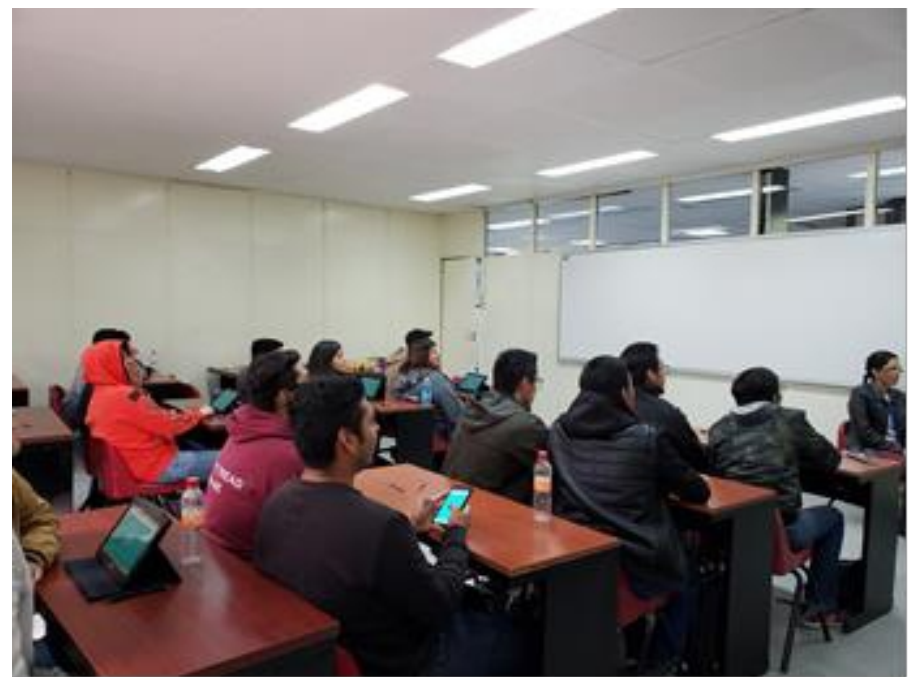

Figura 6. Alumnos entusiastas revisando el encuadre teórico de dinámica de fluidos 